\title{
Medical Decision Support and Medical Informatics Education: Roots, Methods and Applications in Czechoslovakia and the Czech Republic
}

\author{
Jana Zvárová \\ European Center for Medical Informatics, Statistics and Epidemiology of Charles University and \\ Academy of Sciences of the Czech Republic \\ Charles University in Prague, 1st Faculty of Medicine, Institute of Hygiene and Epidemiology \\ Institute of Computer Science of the Academy of Sciences of the Czech Republic, Department of \\ Medical Informatics and Biostatistics \\ Prague, Czech Republic
}

\section{Summary}

The paper describes the history of medical informatics in Czechoslovakia and the Czech Republic. It focuses on the topics of medical informatics education and decision support methods and systems. Several conferences held in Czechoslovakia and in the Czech Republic organized in cooperation with IMIA or EFMI are described. Support of European Union and Gech agencies in several European and national projects focused on medical informatics topics highly contributed to medical informatics development in Czechoslovakia and the Czech Republic and to the establishment of the European Center for Medical Informatics, Statistics and Epidemiology as the joint workplace of Charles University in Prague and Academy of Sciences of the Czech Republic in 1994.

\section{Keywords}

Medical Informatics, history, decision support, education

Yearb Med Inform 2013:206-12

\section{Introduction}

The International Federation for Information Processing (IFIP) is a non-governmental, non-profit umbrella organization for national societies working in the field of information processing. It was established in 1960 as a result of the first World Computer Congress held in Paris in 1959. IFIP has developed its activities through so-called technical committees (TC). The IFIP-TC4 was founded by François Grémy, from France, in 1967. The first interactions of Czechoslovakia with the field of medical informatics started at this time. In the late sixties, the Medical Cybernetics Unit was established at the Institute of Hematology and Blood Transfusion in Prague. One of the three groups of the Medical Cybernetics Unit was focused on research on computer-supported medical decision making, pattern recognition, and health services evaluation. This group organized the first conference Decision processes in clinical medicine in Prague in 1972. This group's research progress was to the great merit of Albert Perez, member of IFIP, who was a leader of the Czech school of information theory. Albert Perez endorsed two of his Ph.D. students to be representatives to the IFIP-TC4 on medical informatics topics. The International Medical Informatics Association (IMIA) grew out of the former TC 4 of the IFIP in 1979. Francois Grémy and Peter Reichertz from Germany deserve credit for the spread of the term medical informatics all over the world.
In Czechoslovakia, the research work of the Medical Cybernetics Unit was stopped as a result of an administrative decision in 1977. The need to pursue the research work and discuss remaining problems lead the researchers of the former Medical Cybernetics Unit to create the Medical Informatics Section in the Czechoslovak Society of Biomedical Engineering in 1979.

\section{The Birth of Medical Informatics (1970 - 1989)}

In the early 1970s, new developments brought first prototypes of decision support systems in medicine and health care to Czechoslovakia. In 1980s, new information and knowledge in the field of medical informatics was acquired by Czechoslovakian researchers mainly through MEDINFO and MIE conferences and through some national conferences with international participation described further in more detail.

\subsection{The 1981 National Conference with International Participation on Medical Informatics}

The national conference Medical Informatics was organized by the Medical Informatics section of the Czechoslovak Society of Biomedical Engineering on 26-28 August, 1981, in Prague. The conference gathered more 
than 100 Czech and Slovak participants. There were three invited lectures given by Peter Adlassnig from Austria, Paul Chastang from France, and by the IMIA President Jan Roukens from the Netherlands.

\subsection{IFIP-IMIA International Work- ing Conference on Computer-Aided Medical Decision Making}

During the MEDINFO 1983 congress in Amsterdam, Jana Zvárová discussed with Jan van Bemmel the intention of the Czechoslovak Society of Biomedical Engineering to organize an IMIA working conference in Prague. Jan van Bemmel strongly supported this idea and promised to chair the program committee. The first IMIA conference held in a socialist country was organized in Prague, Czechoslovakia from September $30^{\text {th }}$ to October $4^{\text {th }} 1985$. Figure 1 shows Jan van Bemmel, chair of the Scientific Program Committee (SPC), Jana Zvarova chair of the Local Organizing Committee (LOC), and Shigekoto Kaihara (then IMIA president) from Japan. The proceedings made of selected full papers, titled Diagnostic Strategies and Expert Systems, were published by Elsevier, North Holland [1]. Among 79 published papers in the proceedings, 30 papers were from Czechoslovakian authors.

\subsection{The 1988 National Conference Computers with International Par- ticipation on Medical Education}

The conference was organized by Charles University in Prague on their premises in August 29-31, 1988. The languages used at the conference were Czech, Slovak, English, and Russian. The papers of the conference focused on using computers in teaching biophysics, physiology, and clinical disciplines, on medical informatics education, and on the use of expert systems. The international speakers were F. Grémy (France), S.A. Simbircev (Union of Soviet Socialist Republics), H. Weihrauchand and J. Michel (German Democratic Republic), R. Engelbrecht (Federal Republic of Germany), J. Doroczewski (Polish People Republic), and G. Mihalas (Romanian Socialist Republic).

\section{The Childhood of Medical Informatics (1990-1999)}

The Velvet Revolution in Czechoslovakia, initiated by a students' strike on November $17^{\text {th }}, 1989$, opened the door for cooperation in science and education with a number of western countries. In 1990s, medical informatics topics were further developed in education and decision support tasks at the national level and through international co-operations.

\subsection{IMIA Working Conference in Prague 1990}

The second IMIA conference held in Prague, 3-7. September 1990, focused on medical informatics and medical education (Figure 2). It brought together participants from 18 countries from all over the world and the proceedings, titled Knowledge, Information and Medical Education, were published by Elsevier in 1991. The proceedings contained over 60 contributions describing the influence of computers on medical education (in the curriculum and at the postgraduate level) and the education in medical informatics in general [2].

\subsection{Education in the Methodology Field of Healthcare}

Due to political changes in the last decade of the twentieth century, Central and Eastern European countries were able to receive support through selected programs of the European Union. The first European project focused on medical informatics education, titled Education in the Methodology Field of Healthcare - EuroMISE (European education in Medical Informatics, Statistics and Epidemiology) was operational from 1993 to 1995. The key issues of this educational programme were to promote multidisciplinary approaches and to focus on healthcare issues that received insufficient attention in traditional training $[3,4]$. The project developed educational programs and designed courses in a broad European co-operation focusing on teaching teachers and health professionals from Central and Eastern European countries. EuroMISE courses given in English consisted of 200 hours of teaching, and more specifically, 160 hours of direct teaching (lectures and practices) and 40 hours devoted to the preparation of the lectures presented by students at EuroMISE workshops and conferences. Moreover, students were given three months to prepare a final thesis on a subject taught during EuroMISE courses.

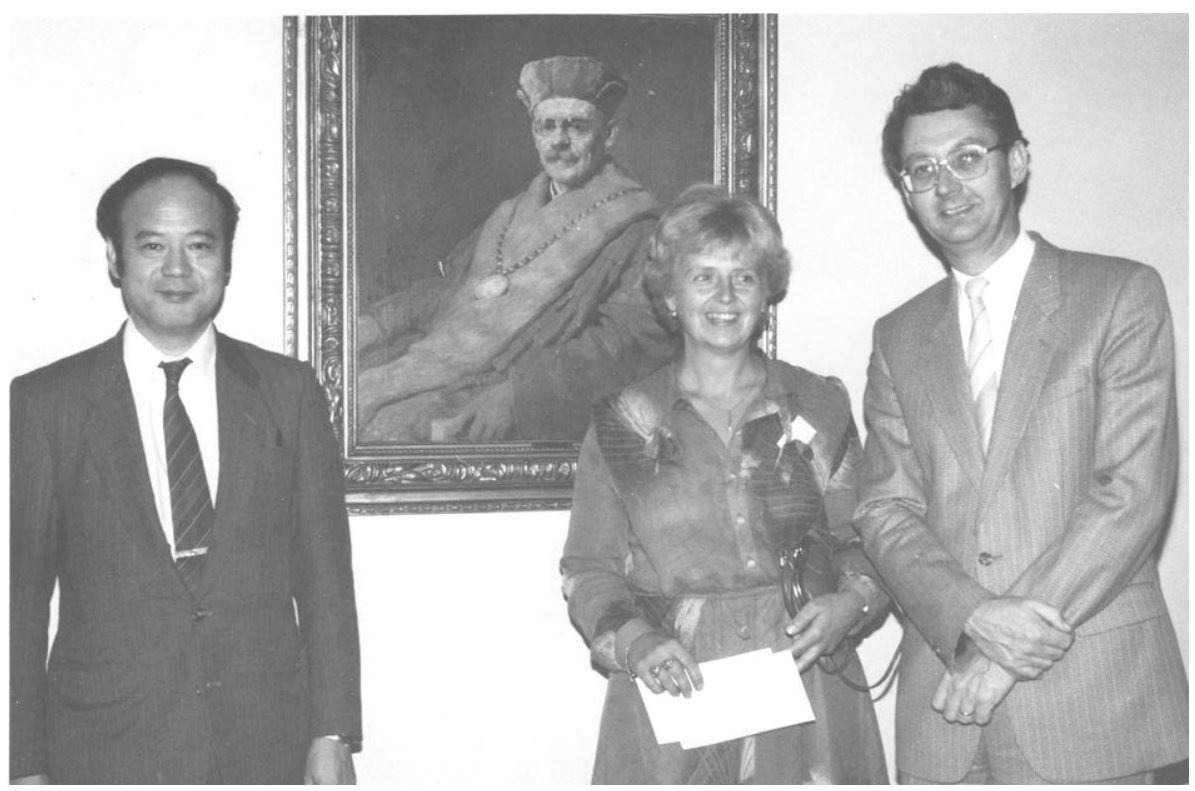

Fig. 1 Shigekoto Kaihara with chairs of the LOC and SPC at the IMIA conference in Prague, 1985. 


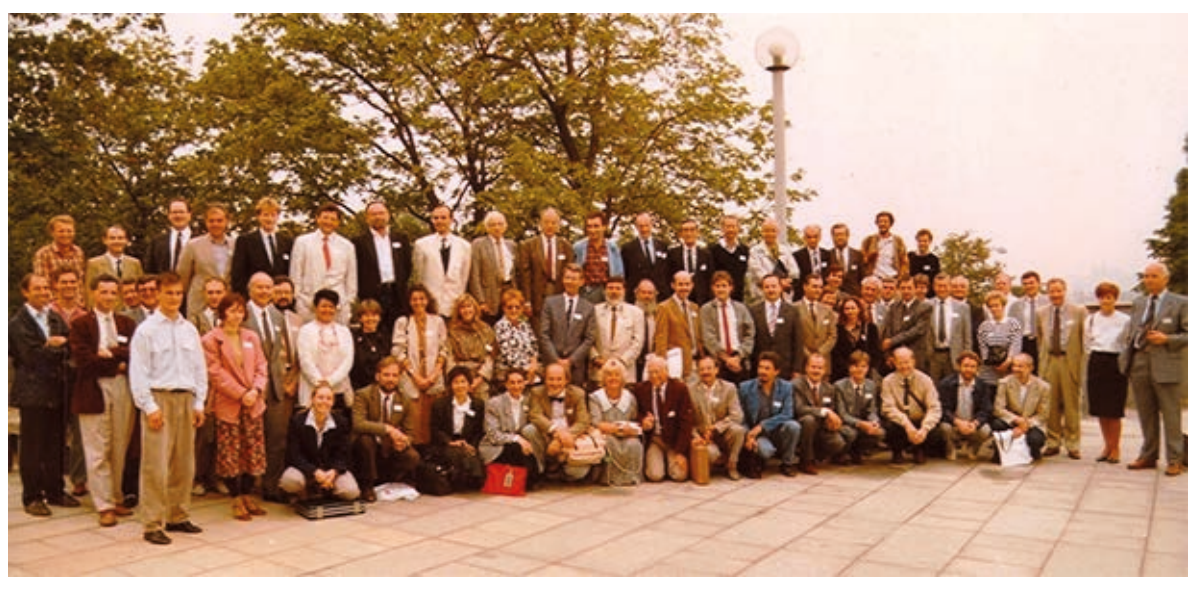

Fig. 2 Participants of the IMIA Working Conference in Prague, 1990.

Czech participants had a sound experience during visits at universities participating in the project. Visits were organized by cooperating EU teachers. In the field of medical informatics, several visits were organized by J.H.van Bemmel and A. Hasman (The Netherlands), R. Engelbrecht, and R.Haux (Germany), and B. Richards (United Kingdom). Participants of EuroMISE courses were able to discover new methods of teaching medical informatics, especially in cooperation with universities, e.g. medical informatics courses developed by the University of Heidelberg (R. Haux) and Heilbronn (H. Dickhaus). The last conference organized in frame of the EuroMISE project was the conference Information, Health and Education, held in Prague, October 20-23, 1995. Among delegates of the conference, there were more than 70 participants from the Czech Republic and other Central and Eastern European countries, who successfully completed the EuroMISE courses. These participants received a Certificate during the Ceremonial session in the Aula Magna of Charles University in Prague.

\subsection{Managing Uncertainty in Medicine}

The second European project entitled Managing Uncertainty in Medicine with the acronym MUM, was operating from 1994 to 1996 . The goal was to contribute to the emerging unification theory of managing uncertainty, when both probabilities and logical approaches were applied, and incorporate these methods into diagnostic knowledge-based systems and smart systems for data analysis. Another important part was to gain practical experience in the field of medical data analysis and knowledge processing by introducing software tools in selected medical health care facilities and by developing decision support systems to be used in various health care facilities in order to offer better treatment to patients, especially in situations where clinical experience may be lacking.

\subsection{European Center for Medical Informatics, Statistics and Epidemiology (EuroMISE Center)}

Both EuroMISE and MUM projects contributed to the founding of the joint programme of the Charles University in Prague and the Academy of Sciences of the Czech Republic named the European Center for Medical Informatics, Statistics and Epidemiology (EuroMISE Center) on 12th April 1994. At the occasion of the EuroMISE Center founding, a one-week conference Medical Informatics Education and Research was held in Harrachov in the Czech Republic. The EuroMISE Center started its joint activities among the Faculty of Mathematics and Physics of Charles University, the First Faculty of Medicine of Charles University, and members of the Institute of Computer Science of the Academy of Sciences of the Czech Republic. In the following years, the activities of the EuroMISE Center were significantly supported by several European grants and during the years 2000-2004 by the national program of research centers of the Czech Republic of the Ministry of Education., Youth and Sports. This support allowed the extension of the multidisciplinary cooperation of the EuroMISE Center to three more institutions, namely the University of Economic in Prague, the University General Hospital in Prague, and the Municipal Hospital in Caslav. An intensive collaboration between the Faculty of Science and the Second Faculty of Medicine of the Charles University was formalized.

\subsection{Information Technology for Education and Training}

The main goal of the 4th framework program of European countries titled IT EDUCTRA (Information Technology EDUcation and TRAining) operating from 1996 to 1998 was to contribute to the information distribution from of information technology in education and training in medicine. Twenty universities and research workplaces and companies took part in the project, among them the EuroMISE Center. One aim was to develop teaching materials in different European languages (including the Czech language) from research results reached in Spain, Germany, Italy, Denmark, Great Britain or other countries. High priority was given to topics such as electronic communication, patient electronic records, safety and security of data, computer-assisted diagnosis, and management of health care quality. Based on the analysis of educational needs in medicine, ten topics were selected for health care education, each of them covering many incremental subjects and disseminated on a $\mathrm{CD}[5]$.

\subsection{EuroMISE Courses from 1996 to 1999}

The financial support given for the EuroMISE project stopped December $31^{\text {st }}$ 1995. Nevertheless, courses were conducted in Prague until the year 1999 due to the personal support of many teachers from organizations participating in the EuroMISE 
project and the personal involvement of other medical informatics colleagues, like Jean Raoul Scherrer from Switzerland and Ioana Moisil from Romania. Between 1996 and 1999, the number of countries and teachers increased and the EuroMISE Center welcomed in Prague students not only coming from Central and Eastern European countries but also from Belgium, Ireland, Turkey, and the United Kingdom. Therefore, through EuroMISE courses, about 150 participants graduated in total. In 1999, a small conference was held at the occasion of the $5^{\text {th }}$ anniversary of the EuroMISE Center. European cooperation stimulated the development of courses and teaching materials in Czech language for a broad dissemination in graduate, postgraduate, and life-long education in the Czech Republic.

\subsection{Integration and Communication for the Continuity of Cardiac Care}

The Integration and Communication for the Continuity of Cardiac Care (I4C) European project was further developed in Czech and Slovak languages as a TripleC Project. It was undertaken between 1998 and 2000 in three hospitals of Eastern Europe (in Prague, Ćáslav, and Bratislava). The goals of the TripleC Project were to test and further develop methods proposed in the I4C Project to design the ORCA (Open Record for Care) Electronic Health Record (EHR) applied to cardiology [6,7]. This project led to the creation of the first structured EHR in both Czech and Slovak languages and evidenced the importance of structuring the information to allow systems interoperability in health care.

The knowledge acquired from the TripleC Project was further advanced in the national research centers project EuroMISE-Cardio (2000-2004), where multidisciplinary research in the field of EHR and decision support systems was conducted with a focus on cardiology and dentistry. The priority was to propose and develop appropriate tools for structured data entry, representation, and processing in order to minimize the effort of users (physicians, nurses) and maximize the clinical relevance of collected data. The suggested Czech solution was implement- ed in a pilot application named MUDR (MUltimedia Distributed electronic health Record) $[8,9]$. Two cardiology outpatient clinics were involved in gathering patients' data and validating research prototypes. The Minimum Data Model of a Cardiology Patient was proposed and research in formalizing clinical guidelines in cardiology was initiated. A voice-recognition component of an EHR used in dental medicine, titled DentCross, was developed and semantic interoperability in cardiology was studied. The synergy of both voice recognition and the graphical representation of data made activities in the dental practice easier, quicker, and more comfortable $[10,11]$. Since 2004, a dental EHR with the interactive voice-recognition DentCross component has been in use in dental care at the University Hospital in Prague-Motol.

\subsection{Medical Guideline Technology}

The MGT (Medical Guideline Technology) European project was conducted during the years 1998 to 2000 . The project focused on the development of computerized tools providing patient-specific practice guidelines (combining general guidelines for specific medical circumstances and specific conditions with a valid price model). Three main applications were created in the same medical domain of hypertension and cardiology by countries that differed in their health care systems, available resources, and organizational structures.

\section{The Adolescence of Medical Informatics (2000-2013)}

Central and Eastern European (CEE) countries made many efforts to be included in the main research and development activities conducted in Europe in the field of medical informatics. CEE countries, including EU CEE countries operate in different environments in terms of economic transformation and status, as well as health care systems. The development of electronic healthcare in the Czech republic was significantly accelerated by education and a broadened research and development in co-operation with all EU countries in the field of medical informatics.

\subsection{Ph.D. Studies in Biomedical Informatics at Charles University in Prague}

The agreement of cooperation of the Charles University in Prague and the Academy of Sciences of the Czech Republic on the doctoral degree study programs in biomedicine was signed on April $23^{\text {rd }}$, 1997. There are now 20 boards of scientific disciplines in postgraduate doctoral studies in biomedicine at Charles University in Prague. As a result of the initiative of the First Faculty of Medicine, in cooperation with the EuroMISE Center, the scientific board of Biomedical Informatics was established in 2001 [12]. Courses were given in Czech and English languages. Doctoral degree study programs are provided either as fulltime programs or in a combined form. The full-time program lasts four years and the combined form can last a maximum of nine years. The requirements for the successful completion of the education program leading to the Ph.D. degree are to pass the National doctoral examination in a chosen field and to defend a thesis, compiled on the basis of personal papers published in peer-reviewed journals.

IMIA Recommendations on Education in Health and Medical Informatics updated in a second version published in Methods of Information in Medicine [13] have been a valuable material for medical informatics education at the Ph.D. level. Students of the Board of Biomedical Informatics are also active in workshops of their specific research field, which are held at the First Faculty of Medicine of Charles University in Prague.

In 2011 and 2012, discussions during workshops were enriched by the questions and comments of the foreign chairs of the workshops B. Blobel (2011) and A. Hasman (2012). In biomedical informatics courses designed for doctoral degree studies, we regularly use interactive electronic books, video films, and audio presentations of lectures. Based on the knowledge gathered 


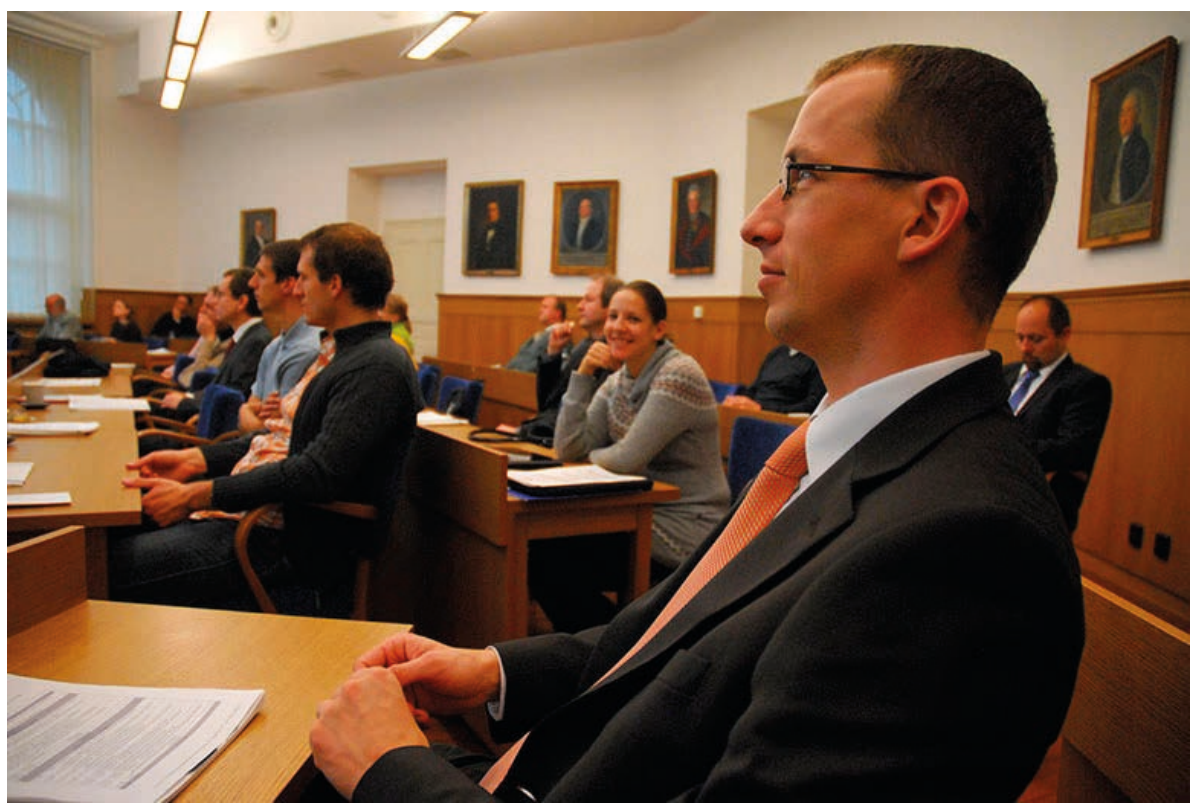

Fig. 3 Workshop of Ph.D. students at the 1st Faculty of Medicine of Charles University in Prague, 2011.

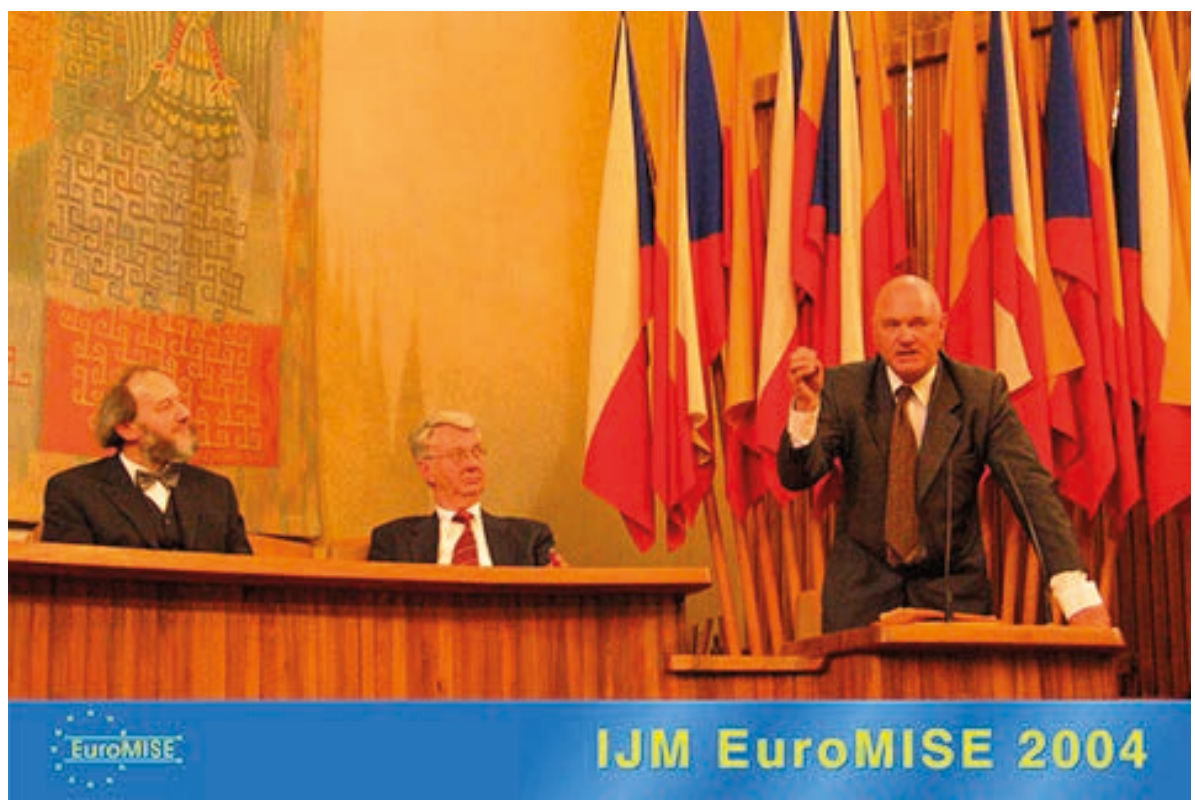

Fig. 4 J.C. Healy from the European Commission introduces eHealth concept.

from the European projects, the EuroMISE Centre has started to develop two editions titled Biomedical Informatics and Biomedical Statistics published in Czech language. Five volumes have been published to date in the Biomedical Informatics edition and three volumes in the Biomedical Statistics edition. Both editions have been published by the Carolinum Printing House of
Charles University in Prague. Since 1998, the ExaMe system is being developed to evaluate students on a targeted knowledge [14]. The system is based on generalized multiple-choice questions. There is no prior restriction on the number of offered answers, but at least one answer is correct and at least one is wrong.. The new research how to evaluate students' knowledge using the
ExaME system can be found in the paper by Martinková.et al. [15] and in the book by Dostálová et al. [16].

\subsection{International Joint Meeting EuroMISE 2004}

Between 12 and 16 April 2004 more than 200 experts from 30 countries gathered in Prague, Czech Republic, to participate to the International Joint Meeting EuroMISE 2004, which consisted of the IMIA working conference Statistical Methodology in Bioinformatics and Clinical Trials and three parallel symposia. The first symposium, organized in cooperation with EFMI focused on Electronic Health Record, Healthcare Registers and Telemedicine, the second symposium centered on Computerized Guidelines and Protocols and the third symposium, held at the $10^{\text {th }}$ anniversary of the EuroMISE Centre, focused on Biomedical Informatics and Biomedical Statistics Education. Moreover, one workshop focused on RTD potential of Central and Eastern Europe for building Information Society in Healthcare and another workshop was on HL7 Standards. Detailed information about EuroMISE 2004 conferences, satellite symposia, and workshops were published in special issues of the International Journal of Medical Informatics [17], [18], and Methods of Information in Medicine [19], [20]. Keynote speeches were given in the Aula Magna of the Charles University in Prague with great impact on the audience (Figure 4).

\subsection{Information Technology for the Development of Continuous Shared Health Care}

The Information Society program of the Academy of Sciences of the Czech Republic was operating from 2004 to 2008. It's theme was the development of methods and tools for providing shared health care continuity in the information society. Research focused on the remote access to information either as data or knowledge from medical records using HL7 standards. It was validated in co-operation with the Municipal Hospital in Časlav. 


\subsection{Center of Biomedical Informatics}

The Center for Biomedical Informatics was operated during the period from 1st March 2006 -to 31st December 2011 with the support of the Czech Research Centers program of the Ministry of Education, Youth and Sports of the Czech Republic as the joint work environment for five organizations. This project had the following main goals a) development of a protocol using an optimal set of genes for the diagnosis and prognosis of cardiovascular diseases, b) design of decision support systems for the diagnosis and personalization of health care in cardiology, c) design of decision support systems for the diagnosis, therapy, and prognosis in dentistry and d) enhancement of quality in postgraduate Ph.D. education and training in the field of biomedical informatics. The research was successfully completed and results were made accessible as papers published in peer-reviewed journals, presentations made during prestigious international conferences, as prototypes of software systems, or as proven technologies, patents, utility patterns, and other outputs. Based on the results of the EuroMISE Center and of the Center of Biomedical Informatics, the platform for multidisciplinary research and higher education was created [21].

\section{Medical Informatics Is Getting Mature}

The importance of information in medicine continues to rise. Medical research and practice benefit from fast development of information and communication technologies. Challenges that medicine is now facing will not be met without access to even more expansive and higher-quality data. Medical applications based on information technology are sure to have a very strong potential to maintain or lower medical costs, improve quality, and support new medical agenda. Many eHealth applications support the processes of health care. It is expected that an increasing development of eHealth applications will influence also medical informatics education and training. Education is the core of the research and development. It is

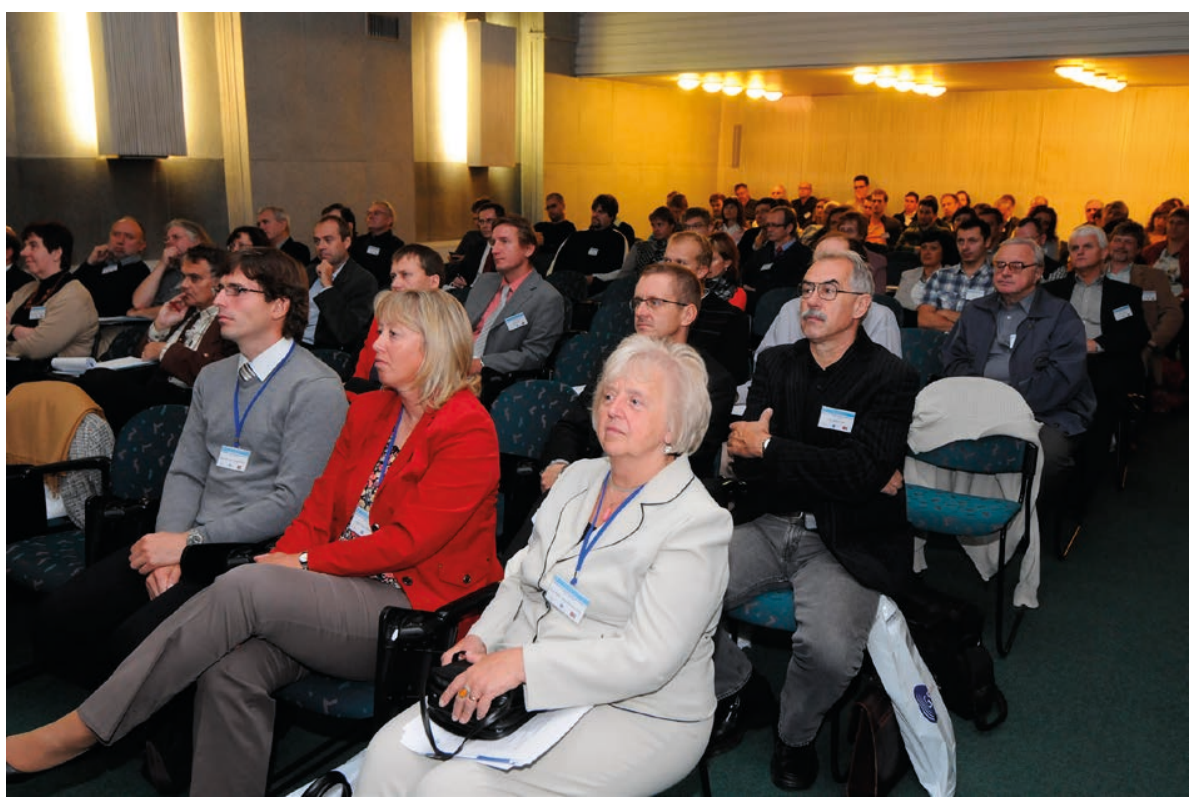

Fig. 5 Participants of the workshop Standards and Electronic Healthcare in 2011.

true for all business branches and the same holds for the health care industry. Since the year 2000, the Czech Society of Biomedical Engineering and Medical Informatics (often in co-operation with the EuroMISE Center) organized at least one workshop per year in the House of Physicians dedicated to a selected medical informatics topic. The workshop in 2001 titled International Standard HL7 - a Pathway to Better Healthcare chaired by W. Ed Hammond from the Duke University in Durham, USA, introduced the HL7 organization and its activities. This workshop initiated the foundation of the HL7 group of the Czech Republic [22]. The workshops in the House of Physicians introduced different topics to a broad audience of Czech physicians, engineers, and other healthcare workers and served also the dissemination of medical informatics knowledge to Ph.D. students. Figure 5 shows the participants of the workshop on Standards and Electronic Healthcare supported by GS $1^{1}$ Czech Republic and held in the House of Physicians in October 2011.

GS1 is an international not-for-profit association with member organizations in over 100 countries. GS1 is dedicated to the design and implementation of global standards and solutions to improve the efficiency and visibility of supply and demand chains globally and across sectors. The GS1 system of standards is the most widely used supply chain standards system in the world.
Medical informatics education has been incorporated to faculty curricula at several universities in the Czech Republic, e.g. Charles University in Prague, Czech Technical University in Prague, Masaryk University in Brno, and Technical University in Brno. The project MEFANET (MEdical FacultiesNETwork) has been operating since 2007 and focuses on the progress in education of medical and health care disciplines using modern information and communication technologies. MEFANET aims at developing and strengthening the cooperation between Czech and Slovak medical faculties. More details about medical informatics education at medical faculties can be found in the web page of MEFANET [23] and in [24, 25, 26].

\section{Conclusion}

Since the foundation of IFIP-TC4 in 1967, the medical informatics field has grown significantly. There are now many journals and books devoted to the field. Of note is the survey initiated by Izet Masic regarding the journals issued in European countries that are members of EFMI [27]. The European Journal for Biomedical Informatics that considers European's needs to share electronic information in the multilingual and 
multicultural European area is published in the Czech Republic [28]. Further of note is the symposium on Biomedical Informatics: Confluence of Multiple Disciplines organized in Heidelberg June 11th 2011 at the occasion of the $50^{\text {th }}$ anniversary of Methods of Information in Medicine [29].

However, there is still a lot of work ahead of us. First, we have to be clear on how we define the term informatics. Do we understand informatics as a computer science or do we consider it as a discipline focused on the information analyzed by different information sciences [30-35]. Second, medical informatics started with a focus on medicine. Nowadays, medical informatics is growing and medical informatics research and education address both biomedicine and healthcare. The medical informatics field has grown to a broad field including biomedical and healthcare informatics. Healthcare informatics is focusing mainly on electronic healthcare development in different countries. Therefore it is focused on the eHealth concept [36] with healthcare applications to be judged not only from electronic but also from the economic and environmental points of view [37]. The environmental view is considered at large, including culture, legislation, language, geographic position, healthcare, and social systems.

More than 40 years after the first national conference on medical decision support in Prague in 1972, we evaluated the development of the medical informatics field at the EFMI special topic conference Data and Knowledge for Medical Decision Support held in Prague, in April 2013 [38]. Many research results from the medical informatics field are deployed in healthcare practice and support the forthcoming electronic healthcare.

\section{Acknowledgment}

The work was partially supported by the project PRVOUK P28/LF1/6.

\section{References}

1. van Bemmel JH, Gremy F, Zvarova J, editors. Diagnostic Strategies and Expert Systems. Amsterdam: North Holland; 1985.

2. van Bemmel JH, Zvarova J, editors. Knowledge, Information and Medical Education. Amsterdam: North Holland; 1991.
3. Zvárová J. Education in Methodology for Health Care-EuroMISE. Methods Inf Med 1994;33:315-7.

4. Zvárová J, Engelbrecht R, van Bemmel JH. Education and training in medical informatics, statistics and epidemiology in EuroMISE. Int J Med Inform 1997;45: 3-8.

5. IT EDUCTRA CD. Commission of the European Communities; 1998.

6. van Ginneken AM, Stam H, van Mulligen EM, de Wilde M, van Mastrigt R, van Bemmel JH. ORCA: the versatile CPR. Methods Inf Med 1999;38:332-8.

7. van Bemmel JH, van Ginneken MA, Stam H, van Mulligen E. Integration and communication for the continuity of cardiac care. J Electrocardiol 1998:31:61-8.

8. Spidlen J., Hanzlicek P., Riha A., Zvarova J. Flexible Information Storage in MUDR EHR. Int J Med Inform 2006;75:201-8.

9. Hanzlicek P, Spidlen J, Heroutova H, Nagy M. User Interface of MUDR Electronic Health Record. Int J Med Inform 2005;74: 221-7.

10. Zvarova J, Dostalova T, Hanzlicek P, Teuberova Z, Nagy M, Pies M, et al. Electronic health record for forensic dentistry. Methods Inf Med 2008,47: 8-13.

11. Hippmann R, Dostálová T, Zvárová J, Nagy M, Seydlova M, Hanzlícek P, et al. Voice-supported Electronic Health Record for Temporomandibular Joint Disorders Methods Inf Med 2010;49(2):168-72.

12. Zvarova J, Svacina S. New Czech Postgraduate Doctoral Program in Biomedical Informatics In: Surjan G, Engelbrecht R, McNair P, editors. Health Data in the Information Society, Proceedings of MIE2002. Amsterdam: IOS Press; 2002. P. 766-9.

13. Mantas J, Ammenwerth E, Demiris G, Hasman A, Haux R, Hersh W, et al. Recommendations of the International Medical Informatics Association (IMIA) on Education in Biomedical and Health Informatics, First Revision. Methods Inf Med 2010;49(2): 105-20.

14. Zvarova J, Zvara K. Evaluation of knowledge using ExaMe program on the internet. In: Iakovidis I, Maglaveras N, Trakatellis A, editors. User acceptance of health telematics applications. Amsterdam: IOS Press; 2000. p. 145-51.

15. Martinková P, Zvára jr., K, Zvárová J, Zvára K. The New Features of the Exame Evaluation System and Reliability of Its Fixed Tests. Methods Inf Med 2006 45:310-5.

16. Dostalova T, Seydlova M. et al. Dentistry and Oral Diseases. Prague: Grada; 2010.

17. Zvárová J, Blobel B. Electronic Health Record Healthcare Registers and Telemedicine. Int J Med Inform 2006;3(75):183-252.

18. Zvárová J, Paralič J. International Joint Meeting EuroMISE 2004 Satellite Workshop on "RTD Potential in Central and Eastern Europe for Building Information Society in Healthcare". Int J Med Inform 2006;4(75):255-342.

19. Zvárová J, Kulikowski C, Mansmann U. Statistical Methodology in Bioinformatics and Clinical Trials. Methods Inf Med 2006;45:137-233.

20. Zvárová J, van Bemmel JH, Mc Cray A. Biomedical Informatics and Biomedical Statistics Education. Methods Inf Med 2006;45:289-321.

21. http://www.cittadella.cz/cbi2012/index.php?p=index\&site $=$ en (last access January 10th, 2013)

22. http://www.hl7.cz/cz/info/news.html (last access
January 10th, 2013)

23. http://www.mefanet.cz/ and http://www.cittadella. cz/cbi2012/index.php?p=index\&site=en (last access January 10th, 2013)

24. Zvarova J, Svacina S, Dostalova T, Seydlova M, Zvara K, Papikova V, et al. Biomedical Informatics Education at Charles University in Prague for Undergraduate and Doctoral Degree Studies. European Journal for Biomedical Informatics 2011,7(2):72-8

25. Svacina S, Zvarova J, editors. Semantic Interoperability in Biomedicine and Healthcare. European Journal for Biomedical Informatics 2011;7:1-74.

26. Zvarova J, Svačina Š, Rauch J, Haase J, Grunfeldová H. EuroMISE Center: Research and Education in Biomedical and Healthcare Informatics. In: Blobel B, Engelbrecht R, Shifrin MA, editors. Large Scale Projects in eHealth. Amsterdam: IOS Press; 2012. p. 53-6

27. Masic I, Kern J, Zvarova J, de Lusignan S. Task force of the EFMI journals: background, rationale and purpose. Stud Health Technol Inform 2009;150:94

28. www.ejbi.org (last access January 10th 2013)

29. Haux R. On the methodology and Scientific Fundamentals of Organizing Representing and Analyzing Data, Information and Knowledge in Biomedicine and Health Care. Methods Inf Med 2011;50 (6):487- 90.

30. van Bemmel JH. The structure of medical informatics. Med Inform (Lond). 1984;9(3-4):175-80.

31. Gremy F. Crisis of Meaning and Medical Informatics Education: A burden and/or a relief? Methods Inf Med 1989;28(4):189-95.

32. Moehr JR. Teaching medical informatics: Teaching on the seams of disciplines, cultures, traditions. Method Inf Med 1989;28:273-80.

33. Zvárová J. On the medical informatics structure. Int J Med Inform 1997;44:75- 81.

34. Zvárová J, Veselý A, Vajda I. Data, Information and Knowledge. In: Berka P, Rauch J, Zighed D. editors. Data Mining and Medical Knowledge Management. Information Science Reference. Hershey, PA: IGI Global; 2009. p. 1-36.

35. Ball M. Medical informatics - back to the future. Keynote lecture MEDINFO 2010, Cape Town 2010.

36. Healy JC. The WHO eHealth resolution - eHealth for all by 2015? Methods Inf Med 2007,46:2-5.

37. Zvarova J, Zvara K. e3Health: Three Main Features of Modern Healthcare. In: Moumtzoglou A, Kastania A. editors. E-Health Systems Quality and Reliability: Models and Standards, Information Science Reference, Hershey, PA: IGI Global; 2011. p. 18-27.

38. Blobel B, Hasman A, Zvárová J, editors. Data and Knowledge for Medical Decision Support. Amsterdam: IOS Press; 2013.

Correspondence to:

Prof. Dr. Jana Zvárová

Charles University in Prague

EuroMISE Centre

First Faculty of Medicine

Katerinská 32

CZ 12108 Prague, The Czech Republic

E-mail:zvarova@euromise.cz 